\title{
On the Structure of Octonion Regular Functions
}

\author{
Janne Kauhanen*(i) and Heikki Orelma
}

\begin{abstract}
In this paper, we study octonion regular functions and the structural differences between regular functions in octonion, quaternion, and Clifford analyses.
\end{abstract}

Mathematics Subject Classification. 30G35, 15A63.

Keywords. Octonions, Cauchy-Riemann operators, Regular functions.

\section{Introduction}

In our recent papers $[5,6]$, we started to study octonion algebraic methods in analysis. This work is a continuation of our studies in this fascinating field. Over the years, many results of octonion analysis have been published and studied since the fundamental paper of Dentoni and Sce [2] was published. One thing which has remained unclear to us is what is octonion analysis all about? A consensus has been that octonion, quaternion and Clifford analyses are similar from a theoretical point of view and, maybe for this reason, octonion analysis has received less attention. Our aim is to prove that octonion analysis and Clifford analysis are different theories from the perpective of regular functions. Thus, octonion analysis is a completely independent research topic.

We start by recalling preliminaries of octonions and Clifford numbers and their connections via triality. We define our fundamental function classes, i.e., left-, right- and bi-regular functions. We give chararacterizations for function classes in biaxial quaternion analysis and in Clifford analysis. The classical Riesz system of Stein and Weiss is used as a familiar reference to clearly see the differences.

The topic of this paper is highly technical, but we have tried to write everything as simply as possible. Hopefully we have succeeded in this job.

\footnotetext{
This article is part of the Topical Collection on FTHD 2018, edited by Sirkka-Liisa Eriksson, Yuri M. Grigoriev, Ville Turunen, Franciscus Sommen and Helmut Malonek.
}

*Corresponding author. 
Many questions remain open and the reader may find a lot of open research problems between the lines. We expect to answer some of these questions when the saga continues.

\section{Preliminaries: Octonion and Clifford Algebras}

In this algebraic part of the paper, we first recall briefly the basic definitions and notations related to octonion and Clifford algebras. In the second place, we study their connections in detail. In the whole paper, our principle is to consider the standard orthonormal basis $\left\{e_{0}, e_{1}, \ldots, e_{7}\right\}$ for $\mathbb{R}^{8}$ equipped with multiplications which lead to nonisomorphic algebras. We will denote the octonion product by $e_{i} \circ e_{j}$, and the Clifford product by $e_{i} e_{j}$.

\subsection{Octonions}

The algebra of octonions $\mathbb{O}$ is the non-commutative and non-associative 8dimensional algebra with the basis $\left\{1, e_{1}, \ldots, e_{7}\right\}$, where $e_{0}$ is denoted by 1 (the identity) and omitted whenever clear from the context, and multiplication given by the following table.

\begin{tabular}{lllllllll}
\hline$\circ$ & 1 & $e_{1}$ & $e_{2}$ & $e_{3}$ & $e_{4}$ & $e_{5}$ & $e_{6}$ & $e_{7}$ \\
\hline 1 & 1 & $e_{1}$ & $e_{2}$ & $e_{3}$ & $e_{4}$ & $e_{5}$ & $e_{6}$ & $e_{7}$ \\
$e_{1}$ & $e_{1}$ & -1 & $e_{3}$ & $-e_{2}$ & $e_{5}$ & $-e_{4}$ & $-e_{7}$ & $e_{6}$ \\
$e_{2}$ & $e_{2}$ & $-e_{3}$ & -1 & $e_{1}$ & $e_{6}$ & $e_{7}$ & $-e_{4}$ & $-e_{5}$ \\
$e_{3}$ & $e_{3}$ & $e_{2}$ & $-e_{1}$ & -1 & $e_{7}$ & $-e_{6}$ & $e_{5}$ & $-e_{4}$ \\
$e_{4}$ & $e_{4}$ & $-e_{5}$ & $-e_{6}$ & $-e_{7}$ & -1 & $e_{1}$ & $e_{2}$ & $e_{3}$ \\
$e_{5}$ & $e_{5}$ & $e_{4}$ & $-e_{7}$ & $e_{6}$ & $-e_{1}$ & -1 & $-e_{3}$ & $e_{2}$ \\
$e_{6}$ & $e_{6}$ & $e_{7}$ & $e_{4}$ & $-e_{5}$ & $-e_{2}$ & $e_{3}$ & -1 & $-e_{1}$ \\
$e_{7}$ & $e_{7}$ & $-e_{6}$ & $e_{5}$ & $e_{4}$ & $-e_{3}$ & $-e_{2}$ & $e_{1}$ & -1 \\
\hline
\end{tabular}

Let us point out that there are several ways to define an octonion product such that $e_{0}=1$. Our choice is historically made, justified by tradition, and, for this reason, we may call it the canonical one. However, for instance, Lounesto uses a different multiplication table in his famous book [8].

For $1 \leq i, j \leq 7$ we have

$$
e_{i} \circ e_{i}=e_{i}^{2}=-1, \quad \text { and } \quad e_{i} \circ e_{j}=-e_{j} \circ e_{i} \quad \text { if } i \neq j .
$$

An element $x \in \mathbb{O}$ may be represented in the forms

$$
\begin{aligned}
x & =x_{0}+x_{1} e_{1}+x_{2} e_{2}+x_{3} e_{3}+x_{4} e_{4}+x_{5} e_{5}+x_{6} e_{6}+x_{7} e_{7} \\
& =x_{0}+\underline{x} \\
& =\left(x_{0}+x_{1} e_{1}+x_{2} e_{2}+x_{3} e_{3}\right)+\left(x_{4}+x_{5} e_{1}+x_{6} e_{2}+x_{7} e_{3}\right) \circ e_{4} \\
& =u+v \circ e_{4} \\
& =\left(u_{0}+\underline{u}\right)+\left(v_{0}+\underline{v}\right) \circ e_{4} .
\end{aligned}
$$

Here, $x_{0}, \ldots, x_{7} \in \mathbb{R}, x_{0}$ is the real part, $\underline{x}$ is the vector part, and $u$ and $v \in \mathbb{H}$ are quaternions. The last form is called the quaternion form of an octonion. 
The conjugate of $x$ is denoted and defined by $\bar{x}=x_{0}-\underline{x}$. Furthermore, the product of two octonions can be written as

$$
\begin{aligned}
x \circ y & =\left(x_{0}+\underline{x}\right) \circ\left(y_{0}+\underline{y}\right)=\sum_{i, j=0}^{7} x_{i} y_{j} e_{i} \circ e_{j}=\sum_{i=0}^{7} x_{i} y_{i} e_{i}^{2}+\sum_{\substack{i, j=0 \\
i \neq j}}^{7} x_{i} y_{j} e_{i} \circ e_{j} \\
& =x_{0} y_{0}-\sum_{i=1}^{7} x_{i} y_{i}+x_{0} \sum_{i=1}^{7} y_{i} e_{i}+y_{0} \sum_{i=1}^{7} x_{i} e_{i}+\sum_{\substack{i, j=1 \\
i \neq j}}^{7} x_{i} y_{j} e_{i} \circ e_{j} \\
& =x_{0} y_{0}-\underline{x} \cdot \underline{y}+x_{0} \underline{y}+y_{0} \underline{x}+\underline{x} \times \underline{y},
\end{aligned}
$$

where $\underline{x} \cdot \underline{y}$ is the dot product and $\underline{x} \times \underline{y}$ the cross product of vectors $\underline{x}$ and $\underline{y}$ in $\mathbb{R}^{7}$.

Denote the quaternion forms of the octonions $x$ and $y$ by

$$
\begin{aligned}
& x=\left(u_{0}+\underline{u}\right)+\left(v_{0}+\underline{v}\right) \circ e_{4}, \\
& y=\left(a_{0}+\underline{a}\right)+\left(b_{0}+\underline{b}\right) \circ e_{4} .
\end{aligned}
$$

In Lemma 2.3 we will write the cross product $\underline{x} \times \underline{y}$ of octonion vector parts $\underline{x}$ and $y$ using the classical 3-dimensional cross products of the vector parts $\underline{u}, \underline{v}, \underline{a}$, and $\underline{b}$ of quaternions (see, e.g., $[3,8,10]$ ).

Lemma 2.1. ([5, Lemma 2.10]) Let $u, v \in \mathbb{H}$. Then

$$
\begin{aligned}
e_{4} \circ u & =\bar{u} \circ e_{4}, \\
e_{4} \circ\left(u \circ e_{4}\right) & =-\bar{u}, \\
\left(u \circ e_{4}\right) \circ e_{4} & =-u, \\
u \circ\left(v \circ e_{4}\right) & =(v \circ u) \circ e_{4}, \\
\left(u \circ e_{4}\right) \circ v & =(u \circ \bar{v}) \circ e_{4}, \\
\left(u \circ e_{4}\right) \circ\left(v \circ e_{4}\right) & =-\bar{v} \circ u .
\end{aligned}
$$

Lemma 2.2. If $x, y \in \mathbb{O}$ are written as in (2), then

$$
\begin{aligned}
\underline{u} \times e_{4} & =\underline{u} \circ e_{4}, \\
e_{4} \times \underline{a} & =-\underline{a} \circ e_{4}, \\
\underline{u} \times\left(\underline{b} \circ e_{4}\right) & =-(\underline{u} \times \underline{b}) \circ e_{4}-(\underline{u} \cdot \underline{b}) e_{4}, \\
e_{4} \times\left(\underline{b} \circ e_{4}\right) & =\underline{b}, \\
\left(\underline{v} \circ e_{4}\right) \times \underline{a} & =-(\underline{v} \times \underline{a}) \circ e_{4}+(\underline{a} \cdot \underline{v}) e_{4}, \\
\left(\underline{v} \circ e_{4}\right) \times e_{4} & =-\underline{v}, \\
\left(\underline{v} \circ e_{4}\right) \times\left(\underline{b} \circ e_{4}\right) & =-\underline{v} \times \underline{b} .
\end{aligned}
$$

Proof. On the one hand, the first two equalities are direct consequences of (1). On the other hand, Lemma 2.1 implies

$$
\begin{aligned}
e_{i} \circ\left(e_{j} \circ e_{4}\right) & =\left(e_{j} \circ e_{i}\right) \circ e_{4}, \\
e_{4} \circ\left(e_{j} \circ e_{4}\right) & =e_{j}, \\
\left(e_{i} \circ e_{4}\right) \circ e_{j} & =-\left(e_{i} \circ e_{j}\right) \circ e_{4},
\end{aligned}
$$




$$
\begin{aligned}
\left(e_{i} \circ e_{4}\right) \circ e_{4} & =-e_{i}, \\
\left(e_{i} \circ e_{4}\right) \circ\left(e_{j} \circ e_{4}\right) & =e_{j} \circ e_{i}
\end{aligned}
$$

for $1 \leq i, j \leq 3$. Then

$$
\begin{aligned}
\underline{u} \times\left(\underline{b} \circ e_{4}\right)= & \sum_{i, j=1}^{3} u_{i} b_{j} e_{i} \circ\left(e_{j} \circ e_{4}\right)=\left(\sum_{i, j=1}^{3} u_{i} b_{j} e_{j} \circ e_{i}\right) \circ e_{4} \\
= & \left(-\sum_{\substack{i, j=1 \\
i \neq j}}^{3} u_{i} b_{j} e_{i} \circ e_{j}-\sum_{i=1}^{3} u_{i} b_{i}\right) \circ e_{4}=-(\underline{u} \times \underline{b}) \circ e_{4}-(\underline{u} \cdot \underline{b}) e_{4}, \\
e_{4} \times\left(\underline{b} \circ e_{4}\right)= & \sum_{j=1}^{3} b_{j} e_{4} \circ\left(e_{j} \circ e_{4}\right)=\sum_{j=1}^{3} b_{j} e_{j}=\underline{b}, \\
\left(\underline{v} \circ e_{4}\right) \times \underline{a}= & \sum_{i, j=1}^{3} v_{i} a_{j}\left(e_{i} \circ e_{4}\right) \circ e_{j}=\left(-\sum_{i, j=1}^{3} v_{i} a_{j} e_{i} \circ e_{j}\right) \circ e_{4} \\
= & \left(-\sum_{i, j=1}^{3} v_{i} a_{j} e_{i} \circ e_{j}+\sum_{i=1}^{3} v_{i} a_{i} \circ e_{4}=-(\underline{v} \times \underline{a}) \circ e_{4}+(\underline{a} \cdot \underline{v}) e_{4},\right. \\
\left(\underline{i \neq j} \circ e_{4}\right) \times e_{4}= & \sum_{i=1}^{3} v_{i}\left(e_{i} \circ e_{4}\right) \circ e_{4}=-\sum_{i=1}^{3} v_{i} e_{i}=-\underline{v},
\end{aligned}
$$

and

$$
\begin{aligned}
\left(\underline{v} \circ e_{4}\right) \times\left(\underline{b} \circ e_{4}\right) & =\sum_{\substack{i, j=1 \\
i \neq j}}^{3} v_{i} b_{j}\left(e_{i} \circ e_{4}\right) \circ\left(e_{j} \circ e_{4}\right)=\sum_{\substack{i, j=1 \\
i \neq j}}^{3} v_{i} b_{j} e_{j} \circ e_{i} \\
& =-\sum_{\substack{i, j=1 \\
i \neq j}}^{3} v_{i} b_{j} e_{i} \circ e_{j} \\
& =-\underline{v} \times \underline{b} .
\end{aligned}
$$

Lemma 2.3. Consider the quaternion representations of the vectors $\underline{x}$ and $\underline{y} \in \mathbb{O}$ as in (2). Then the cross product in quaternion form is

$$
\begin{aligned}
\underline{x} \times \underline{y}= & v_{0} \underline{b}-\underline{v} b_{0}+\underline{u} \times \underline{a}-\underline{v} \times \underline{b} & & \in \operatorname{span}\left\{e_{1}, e_{2}, e_{3}\right\} \\
& +(\underline{v} \cdot \underline{a}-\underline{u} \cdot \underline{b}) e_{4} & & \in \operatorname{span}\left\{e_{4}\right\} \\
& +\left(\underline{u} b_{0}-v_{0} \underline{a}-\underline{u} \times \underline{b}-\underline{v} \times \underline{a}\right) \circ e_{4} & & \in \operatorname{span}\left\{e_{5}, e_{6}, e_{7}\right\}
\end{aligned}
$$

Proof. By Lemma 2.2, we obtain

$$
\begin{aligned}
\underline{x} \times \underline{y}= & \underline{u} \times \underline{a}+\underline{u} \times\left(b_{0} e_{4}\right)+\underline{u} \times\left(\underline{b} \circ e_{4}\right) \\
& +\left(v_{0} e_{4}\right) \times \underline{a}+\left(v_{0} e_{4}\right) \times\left(b_{0} e_{4}\right)+\left(v_{0} e_{4}\right) \times\left(\underline{b} \circ e_{4}\right) \\
& +\left(\underline{v} \circ e_{4}\right) \times \underline{a}+\left(\underline{v} \circ e_{4}\right) \times\left(b_{0} e_{4}\right)+\left(\underline{v} \circ e_{4}\right) \times\left(\underline{b} \circ e_{4}\right)
\end{aligned}
$$




$$
\begin{aligned}
= & \underline{u} \times \underline{a}+\underline{u} b_{0} \circ e_{4}-(\underline{u} \times \underline{b}) \circ e_{4} \\
& -v_{0} \underline{a} \circ e_{4}+0+v_{0} \underline{b} \\
& -(\underline{v} \times \underline{a}) \circ e_{4}-\underline{v} b_{0}-\underline{v} \times \underline{b} \\
= & \underline{u} \times \underline{a}-\underline{v} \times \underline{b}+v_{0} \underline{b}-\underline{v} b_{0} \\
& +(\underline{v} \cdot \underline{a}-\underline{u} \cdot \underline{b}) e_{4} \\
& +\left(\underline{u} b_{0}-v_{0} \underline{a}-\underline{u} \times \underline{b}-\underline{v} \times \underline{a}\right) \circ e_{4} .
\end{aligned}
$$

Corollary 2.4. If $x, y \in \mathbb{O}$ are written as in (2), then

$$
\begin{aligned}
x \circ y= & u_{0} a_{0}-v_{0} b_{0}-\underline{u} \cdot \underline{a}-\underline{v} \cdot \underline{b} & & \in \mathbb{R} \\
& +u_{0} \underline{a}+a_{0} \underline{u}+v_{0} \underline{b}-\underline{v} b_{0}+\underline{u} \times \underline{a}-\underline{v} \times \underline{b} & & \in \operatorname{span}\left\{e_{1}, e_{2}, e_{3}\right\} \\
& +\left(u_{0} b_{0}+a_{0} v_{0}+\underline{v} \cdot \underline{a}-\underline{u} \cdot \underline{b}\right) e_{4} & & \in \operatorname{span}\left\{e_{4}\right\} \\
& +\left(u_{0} \underline{b}+a_{0} \underline{v}+\underline{u} b_{0}-v_{0} \underline{a}-\underline{u} \times \underline{b}-\underline{v} \times \underline{a}\right) \circ e_{4} & & \in \operatorname{span}\left\{e_{5}, e_{6}, e_{7}\right\}
\end{aligned}
$$

\subsection{The Clifford Algebra $\mathcal{C} \ell_{0,7}$ and Triality}

Since the dimension of the underlying vector space of the octonions and Clifford paravectors is 8 , they behave similarly as vector spaces. Moreover, we may ask if there is a connection between the octonion product and the Clifford product? The answer is given by Pertti Lounesto in his book [8]. We will recall his ideas here in detail. Let us recall the basic definitions and properties of Clifford algebras.

We continue working with the basis $\left\{e_{0}, e_{1}, \ldots, e_{7}\right\}$ for $\mathbb{R}^{8}$. The Clifford product is defined by

$$
e_{i} e_{j}+e_{j} e_{i}=-2 \delta_{i j}, i, j=1, \ldots, 7,
$$

where $\delta_{i j}$ is the Kronecker delta symbol. Here, $e_{0}=1$. Then, similarly to the case of octonions, $e_{0}^{2}=1$, and $e_{j}^{2}=-1$ for all $j=1, \ldots, 7$. The Clifford product $e_{i} e_{j}$ is not necessarily a vector or a scalar. This product generates an associative algebra, called the Clifford algebra, denoted by $\mathcal{C} \ell_{0,7}$. The dimension of this Clifford algebra is $2^{7}$, and an element $a \in \mathcal{C} \ell_{0,7}$ may be represented as a sum

$$
a=\sum_{j=0}^{7}[a]_{j}
$$

of a scalar part $[a]_{0}$, generated by 1 , a 1 -vector part $[a]_{1}$, generated by $e_{j}$ 's, a 2 -vector part $[a]_{2}$, generated by the products $e_{i} e_{j}$, where $1 \leq i<j \leq 7$, etc. Clifford numbers of the form $[a]_{1}$ are called vectors and those of the form $[a]_{0,1}=[a]_{0}+[a]_{1}$ are called paravectors. The set of paravectors may be identified with $\mathbb{R}^{8}$.

The Clifford product of two paravectors $x$ and $y$ can be written as

$$
\begin{aligned}
x y & =\left(x_{0}+\underline{x}\right)\left(y_{0}+\underline{y}\right)=\sum_{i, j=0}^{7} x_{i} y_{j} e_{i} e_{j} \\
& =\sum_{i=0}^{7} x_{i} y_{i} e_{i}^{2}+\sum_{\substack{i, j=0 \\
i \neq j}}^{7} x_{i} y_{j} e_{i} e_{j}
\end{aligned}
$$




$$
\begin{aligned}
& =x_{0} y_{0}-\sum_{i=1}^{7} x_{i} y_{i}+x_{0} \sum_{i=1}^{7} y_{i} e_{i}+y_{0} \sum_{i=1}^{7} x_{i} e_{i}+\sum_{\substack{i, j=1 \\
i \neq j}}^{7} x_{i} y_{j} e_{i} e_{j} \\
& =x_{0} y_{0}-\underline{x} \cdot \underline{y}+x_{0} \underline{y}+y_{0} \underline{x}+\underline{x} \wedge \underline{y},
\end{aligned}
$$

where $\underline{x} \wedge \underline{y}$ is the wedge product of vectors $\underline{x}$ and $\underline{y}$. In particular, $\underline{x} \underline{y}=$ $\underline{x} \wedge \underline{y}-\underline{x} \cdot \underline{y}$.

The reader can see that formally the octonion and the Clifford products are similar, and a reasonable question is "how they are connected?". We would like to construct the octonion product using the Clifford algebra $\mathcal{C} \ell_{0,7}$. Let us consider the octonion product of the basis elements $e_{i}$ and $e_{j}$, where $1 \leq i, j \leq 7, i \neq j$ :

$$
e_{i} \circ e_{j}=e_{k}
$$

Then $1 \leq k \leq 7$, and $i \neq k \neq j$. The corresponding Clifford product $e_{i} e_{j}$ may be mapped to $e_{k}$ by multiplying it by the trivector $e_{j} e_{i} e_{k}$, i.e.,

$$
\left(e_{i} e_{j}\right)\left(e_{j} e_{i} e_{k}\right)=e_{i}^{2} e_{j}^{2} e_{k}=e_{k} .
$$

Using the same trivector, $e_{j} e_{i}$ is mapped to $-e_{k}$. If $\underline{a}$ and $\underline{b}$ are vectors, then

$$
\underline{a} \underline{b}\left(e_{j} e_{i} e_{k}\right)=\left(a_{i} b_{j}-a_{j} b_{i}\right) e_{k}+\left[\underline{a} \underline{b}\left(e_{j} e_{i} e_{k}\right)\right]_{3}+\left[\underline{a} \underline{b}\left(e_{j} e_{i} e_{k}\right)\right]_{5} .
$$

Picking the 1-vector part

$$
\left[\underline{a} \underline{b}\left(e_{j} e_{i} e_{k}\right)\right]_{1}=\left(a_{i} b_{j}-a_{j} b_{i}\right) e_{k},
$$

we get a part of the $k$ th component of the octonion product $\underline{a} \circ \underline{b}$. Using this idea, we may express the octonion product $a \circ b$ as the paravector part of the Clifford product $a b(1-W)$, where $W$ is a suitable 3 -vector.

Lemma 2.5. ([8, Sec 23.3], [12, Lemma 4.1]) Define

$$
W=e_{123}+e_{145}+e_{176}+e_{246}+e_{257}+e_{347}+e_{365} .
$$

Let $a=a_{0}+\underline{a}$ and $b=b_{0}+\underline{b}$ be paravectors. Then

$$
a \circ b=[a b(1-W)]_{0,1}
$$

and in particular, $\underline{a} \times \underline{b}=-[(\underline{a} \wedge \underline{b}) W]_{1}$.

Lounesto states Lemmas 2.5 and 2.7 without proofs at pages 303-304 in [8]. He uses a different multiplication table of octonions, and therefore the seven basis vectors $e_{i j k}$ have different indices $i, j$, and $k$ in his trivector $W$. Venäläinen gives a proof for Lemma 2.5 in her licentiate thesis [12]. For the convenience of the reader, we give a proof of Lemma 2.5 here.

Proof. To begin with, we have

$$
[a b(1-W)]_{0,1}=[a b]_{0,1}-[a b W]_{1}=a_{0} b_{0}-\underline{a} \cdot \underline{b}+a_{0} \underline{b}+b_{0} \underline{a}-[a b W]_{1} .
$$

By (1) and (3), it is enough to show that $\underline{a} \times \underline{b}=-[(\underline{a} \wedge \underline{b}) W]_{1}$. Consider the triplets

$$
\nu=123,145,176,246,257,347,365 .
$$


The product $e_{i} e_{j} e_{\nu}$ is a vector only if the pair of indices $i j$ belongs to the triplet $\nu$. Since the cross and the wedge products

$$
\underline{a} \times \underline{b}=\sum_{\substack{i, j=1 \\ i<j}}^{7}\left(a_{i} b_{j}-a_{j} b_{i}\right) e_{i} \circ e_{j} \quad \text { and } \quad \underline{a} \wedge \underline{b}=\sum_{\substack{i, j=1 \\ i<j}}^{7}\left(a_{i} b_{j}-a_{j} b_{i}\right) e_{i} e_{j}
$$

have the same coefficients, and each pair $i j, 1 \leq i<j \leq 7$, is contained in exatly one of the triplets $\nu$, say $\nu_{0}$, it is enough to check that $e_{i} e_{j} e_{\nu_{0}}=-e_{i} \circ e_{j}$ for all such pairs $i j$.

A straightforward computation shows:

Lemma 2.6. The trivector $W=e_{123}+e_{145}+e_{176}+e_{246}+e_{257}+e_{347}+e_{365}$ is invertible with

$$
W^{-1}=\frac{1}{7}\left(W-6 e_{12 \cdots 7}\right) .
$$

In the above, we identified octonions $\mathbb{O}$ with the 8-dimensional paravectors. The dimension 8 plays a special role in the theory of spin groups, since Spin(8) has the so called exceptional automorphisms. This feature is called triality, and the first time it was noticed was in the book of Study [11]. For modern references, see $[4,8,10]$. The triality means that in addition to paravectors, we may identify octonions with the spinor spaces $S^{ \pm}$. The spinor spaces may be realized by

$$
S^{ \pm}=\mathcal{C} \ell_{0,7} I^{ \pm}
$$

where $I^{ \pm}$is a primitive idempotent,

$$
I^{ \pm}=\frac{1}{16}\left(1+W e_{12 \cdots 7}\right)\left(1 \pm e_{12 \cdots 7}\right),
$$

see $[1,8]$. A straightforward computation shows that the octonion product in spinor spaces may be determined as follows.

Lemma 2.7. [8, Sec 23.3] For paravectors $a$ and $b$, we have

$$
a \circ b=16\left[a b I^{-}\right]_{0,1} .
$$

\section{Octonion Analysis}

In this section, we recall basic facts of octonion analysis, i.e., the theory of Cauchy-Riemann operators in the octonionic setting. After that, we carefully study the general structure of the null solutions of these operators and define four different classes of regular functions: left-, right-, B-, and R-regular functions. R-regular functions are just solutions of the classical Riesz system. We use the Riesz system here as a familiar reference to better understand the structure of octonion regular functions. In Clifford analysis the corresponding function classes are equal. This structural difference is a fundamental difference between octonion and Clifford analyses. 


\subsection{Cauchy-Riemann Operators}

A function $f: \mathbb{R}^{8} \rightarrow \mathbb{O}$ is of the form $f=f_{0}+f_{1} e_{1}+\cdots+f_{7} e_{7}=f_{0}+\underline{f}$, where $f_{j}: \mathbb{R}^{8} \rightarrow \mathbb{R}$. We define the Cauchy-Riemann operator

$$
D_{x}=\partial_{x_{0}}+e_{1} \circ \partial_{x_{1}}+\cdots+e_{7} \circ \partial_{x_{7}}
$$

Its vector part,

$$
D_{\underline{x}}=e_{1} \circ \partial_{x_{1}}+\cdots+e_{7} \circ \partial_{x_{7}}
$$

is called the Dirac operator. If the coordinate functions of $f$ have partial derivatives, then $D_{x}$ operates on $f$ from the left and from the right as

$$
D_{x} f=\sum_{i, j=0}^{7} e_{i} \circ e_{j} \partial_{x_{i}} f_{j} \quad \text { and } \quad f D_{x}=\sum_{i, j=0}^{7} e_{j} \circ e_{i} \partial_{x_{i}} f_{j}
$$

Decomposition (1) gives (see [7])

$$
\begin{aligned}
& D_{x} f=\partial_{x_{0}} f_{0}-D_{\underline{x}} \cdot \underline{f}+\partial_{x_{0}} \underline{f}+D_{\underline{x}} f_{0}+D_{\underline{x}} \times \underline{f} \quad \text { and } \\
& f D_{x}=\partial_{x_{0}} f_{0}-D_{\underline{x}} \cdot \underline{f}+\partial_{x_{0}} \underline{f}+D_{\underline{x}} f_{0}-D_{\underline{x}} \times \underline{f}
\end{aligned}
$$

where $\partial_{x_{0}} f_{0}-D_{\underline{x}} \cdot \underline{f}$ is the divergence of $f$ and $D_{\underline{x}} \times \underline{f}$ is the rotor of $\underline{f}$.

If $D_{x} f=0$ (resp., $f D_{x}=0$ ), then $f$ is called left (resp., right) regular.

In Clifford analysis one studies functions $f: \mathbb{R}^{8} \rightarrow \mathcal{C} \ell_{0,7}$. We define the Cauchy-Riemann operator similarly as in octonion analysis:

$$
\partial_{x}=\partial_{x_{0}}+e_{1} \partial_{x_{1}}+\cdots+e_{7} \partial_{x_{7}}=\partial_{x_{0}}+\partial_{\underline{x}}
$$

Functions satisfying $\partial_{x} f=0$ (resp., $f \partial_{x}=0$ ) on $\mathbb{R}^{8}$ are called left (resp., right) monogenic. In this paper we only need to consider paravector valued functions

$$
f=f_{0}+f_{1} e_{1}+\cdots+f_{7} e_{7} .
$$

\subsection{Left-, Right-, B- and R-Regular Functions}

Comparing the real and vector parts in (6) and (7) yields the following well known results.

Proposition 3.1. A function $f: \mathbb{R}^{8} \rightarrow \mathbb{O}$ is left regular if and only if it satisfies the Moisil-Teodorescu type system

$$
\begin{aligned}
\partial_{x_{0}} f_{0}-D_{\underline{x}} \cdot \underline{f} & =0, \\
\partial_{x_{0}} \underline{f}+D_{\underline{x}} f_{0}+D_{\underline{x}} \times \underline{f} & =0,
\end{aligned}
$$


whose componentwise form is

$$
\begin{aligned}
\partial_{x_{0}} f_{0}-\partial_{x_{1}} f_{1}-\ldots-\partial_{x_{7}} f_{7}=0 \\
\partial_{x_{0}} f_{1}+\partial_{x_{1}} f_{0}+\partial_{x_{2}} f_{3}-\partial_{x_{3}} f_{2}+\partial_{x_{4}} f_{5}-\partial_{x_{5}} f_{4}-\partial_{x_{6}} f_{7}+\partial_{x_{7}} f_{6}=0, \\
\partial_{x_{0}} f_{2}+\partial_{x_{2}} f_{0}-\partial_{x_{1}} f_{3}+\partial_{x_{3}} f_{1}+\partial_{x_{4}} f_{6}-\partial_{x_{6}} f_{4}+\partial_{x_{5}} f_{7}-\partial_{x_{7}} f_{5}=0, \\
\partial_{x_{0}} f_{3}+\partial_{x_{3}} f_{0}+\partial_{x_{1}} f_{2}-\partial_{x_{2}} f_{1}+\partial_{x_{4}} f_{7}-\partial_{x_{7}} f_{4}-\partial_{x_{5}} f_{6}+\partial_{x_{6}} f_{5}=0, \\
\partial_{x_{0}} f_{4}+\partial_{x_{4}} f_{0}-\partial_{x_{1}} f_{5}+\partial_{x_{5}} f_{1}-\partial_{x_{2}} f_{6}+\partial_{x_{6}} f_{2}-\partial_{x_{3}} f_{7}+\partial_{x_{7}} f_{3}=0, \\
\partial_{x_{0}} f_{5}+\partial_{x_{5}} f_{0}+\partial_{x_{1}} f_{4}-\partial_{x_{4}} f_{1}-\partial_{x_{2}} f_{7}+\partial_{x_{7}} f_{2}+\partial_{x_{3}} f_{6}-\partial_{x_{6}} f_{3}=0 \\
\partial_{x_{0}} f_{6}+\partial_{x_{6}} f_{0}+\partial_{x_{1}} f_{7}-\partial_{x_{7}} f_{1}+\partial_{x_{2}} f_{4}-\partial_{x_{4}} f_{2}-\partial_{x_{3}} f_{5}+\partial_{x_{5}} f_{3}=0, \\
\partial_{x_{0}} f_{7}+\partial_{x_{7}} f_{0}-\partial_{x_{1}} f_{6}+\partial_{x_{6}} f_{1}+\partial_{x_{2}} f_{5}-\partial_{x_{5}} f_{2}+\partial_{x_{3}} f_{4}-\partial_{x_{4}} f_{3}=0 .
\end{aligned}
$$

We will denote the space of left regular functions by $\mathcal{M}^{(\ell)}$, and similary, right regular functions by $\mathcal{M}^{(r)}$.

Proposition 3.2. A function $f: \mathbb{R}^{8} \rightarrow \mathbb{O}$ is both left and right regular if and only if it satisfies the system

$$
\begin{aligned}
\partial_{x_{0}} f_{0}-D_{\underline{x}} \cdot \underline{f} & =0, \\
\partial_{x_{0}} \underline{f}+D_{\underline{x}} f_{0} & =0, \\
D_{\underline{x}} \times \underline{f} & =0,
\end{aligned}
$$

whose componentwise form is

$$
\begin{aligned}
& \partial_{x_{0}} f_{0}-\partial_{x_{1}} f_{1}-\ldots-\partial_{x_{7}} f_{7}=0, \\
& \partial_{x_{0}} f_{i}+\partial_{x_{i}} f_{0}=0, \quad i=1, \ldots, 7, \\
& \partial_{x_{2}} f_{3}-\partial_{x_{3}} f_{2}+\partial_{x_{4}} f_{5}-\partial_{x_{5}} f_{4}-\partial_{x_{6}} f_{7}+\partial_{x_{7}} f_{6}=0, \\
& -\partial_{x_{1}} f_{3}+\partial_{x_{3}} f_{1}+\partial_{x_{4}} f_{6}-\partial_{x_{6}} f_{4}+\partial_{x_{5}} f_{7}-\partial_{x_{7}} f_{5}=0, \\
& \partial_{x_{1}} f_{2}-\partial_{x_{2}} f_{1}+\partial_{x_{4}} f_{7}-\partial_{x_{7}} f_{4}-\partial_{x_{5}} f_{6}+\partial_{x_{6}} f_{5}=0, \\
& -\partial_{x_{1}} f_{5}+\partial_{x_{5}} f_{1}-\partial_{x_{2}} f_{6}+\partial_{x_{6}} f_{2}-\partial_{x_{3}} f_{7}+\partial_{x_{7}} f_{3}=0, \\
& \partial_{x_{1}} f_{4}-\partial_{x_{4}} f_{1}-\partial_{x_{2}} f_{7}+\partial_{x_{7}} f_{2}+\partial_{x_{3}} f_{6}-\partial_{x_{6}} f_{3}=0, \\
& \partial_{x_{1}} f_{7}-\partial_{x_{7}} f_{1}+\partial_{x_{2}} f_{4}-\partial_{x_{4}} f_{2}-\partial_{x_{3}} f_{5}+\partial_{x_{5}} f_{3}=0, \\
& -\partial_{x_{1}} f_{6}+\partial_{x_{6}} f_{1}+\partial_{x_{2}} f_{5}-\partial_{x_{5}} f_{2}+\partial_{x_{3}} f_{4}-\partial_{x_{4}} f_{3}=0 .
\end{aligned}
$$

We will call functions satisfying (11) B-regular, and denote the space of such functions by $\mathcal{M}_{B}$. Naturally

$$
\mathcal{M}_{B}=\mathcal{M}^{(\ell)} \cap \mathcal{M}^{(r)} .
$$

The fundamental difference between octonion and Clifford analyses is that in Clifford analysis the paravector valued null solutions to the CauchyRiemann operator satisfy the Riesz system and are at the same time left and right monogenic, which is not true in octonion analysis. The following well-known proposition follows from the definitions, similarly as in octonion analysis, by comparing the scalar parts, 1-vector parts, and 2-vector parts. 
Proposition 3.3. Suppose $f: \mathbb{R}^{8} \rightarrow \mathcal{C} \ell_{0,7}$ is a paravector valued function. Then $\partial_{x} f=0$ if and only if $f \partial_{x}=0$, and this is equivalent to $f$ satisfying the Riesz-system

$$
\begin{aligned}
\partial_{x_{0}} f_{0}-\partial_{\underline{x}} \cdot \underline{f} & =0, \\
\partial_{x_{0}} \underline{f}+\partial_{\underline{x}} f_{0} & =0, \\
\partial_{\underline{x}} \wedge \underline{f} & =0,
\end{aligned}
$$

whose componentwise form is

$$
\begin{aligned}
\partial_{x_{0}} f_{0}-\partial_{x_{1}} f_{1}-\ldots-\partial_{x_{7}} f_{7} & =0, \\
\partial_{x_{0}} f_{i}+\partial_{x_{i}} f_{0} & =0, \quad i=1, \ldots, 7, \\
\partial_{x_{i}} f_{j}-\partial_{x_{j}} f_{i} & =0, \quad i, j=1, \ldots, 7, i \neq j,
\end{aligned}
$$

Functions satisfying (13) are called $R$-regular, and the space of such functions is denoted by $\mathcal{M}_{R}$.

To convince the reader about the existence of these function classes, we recall the following classical method from Clifford analysis.

Remark 3.4. (Cauchy-Kovalevskaya extension) If $f: \Omega \rightarrow \mathbb{R}$ is a real analytic function defined on an open $\Omega \subset \mathbb{R}^{7} \cong \mathbb{O} \cap\left\{x_{0}=0\right\}$ we may construct its Cauchy-Kovalevskaya extension analogously to Clifford analysis (see [1]) by defining

$$
\mathrm{CK}[f](x)=e^{-x_{0} D_{\underline{x}}} f(\underline{x}) .
$$

It is easy to see that since $f$ is real valued, $D_{x} \mathrm{CK}[f]=\mathrm{CK}[f] D_{x}=0$, i.e., $\mathrm{CK}[f] \in \mathcal{M}_{B}$. Since $\mathbb{O}$ is an alternative division algebra, that is $x(x y)=x^{2} y$ for all $x, y \in \mathbb{O}$, the Cauchy-Kovalevskaya extension may be extended to octonion valued real analytic functions. A necessary condition for $\mathrm{CK}[f] \in$ $\mathcal{M}^{(\ell)}$ is that $f$ is an octonion valued real analytic function with $\underline{f} \neq 0$. This condition is not sufficient since, e.g.,

$$
\mathrm{CK}[\underline{x}](x)=7 x_{0}+\underline{x}
$$

belongs to $\mathcal{M}_{B}$.

We may conclude that although Clifford and octonion analyses have formally very similar definitions, the corresponding function spaces are different.

Proposition 3.5. $\mathcal{M}_{R} \subsetneq \mathcal{M}_{B} \subsetneq \mathcal{M}^{(\ell)}$

Proof. Two inclusions follow from Propositions 3.1-3.3. The examples showing that the inclusions are strict, respectively, are: if $f=x_{2} e_{1}-x_{7} e_{4}$, then $D_{x} f=f D_{x}=0$, but $\partial_{x_{2}} f_{1}-\partial_{x_{1}} f_{2}=1 \neq 0$, and if $f=x_{1}-x_{2} e_{3}$, then $D_{x} f=0$, but $f D_{x}=2 e_{1} \neq 0$.

This result is crucial in understanding the fundamental character of octonion analysis and the structural differences between octonion, quaternion, and Clifford analyses. 
Remark 3.6. (Quaternion analysis) If we make the corresponding definitions for quaternion regular function classes by considering the Cauchy-Riemann operator $D_{x}=\partial_{x_{0}}+e_{1} \circ \partial_{x_{1}}+e_{2} \circ \partial_{x_{2}}+e_{3} \circ \partial_{x_{3}}$ acting on quaternion valued functions $f=f_{1}+f_{1} e_{1}+f_{2} e_{2}+f_{3} e_{3}$, then, by comparing (11) and (13), we observe immediately that

$$
\mathcal{M}_{R}=\mathcal{M}_{B} \subsetneq \mathcal{M}^{(\ell)}
$$

Remark 3.7. (Clifford analysis) If we make the corresponding definitions for paravector valued monogenic functions, then, by Proposition 3.3,

$$
\mathcal{M}_{R}=\mathcal{M}_{B}=\mathcal{M}^{(\ell)} .
$$

\section{Function Classes in Biaxial Quaternion Analysis}

In the preceding section we gave characterizations for left-, $B$-, and $R$-regular functions using componentwise and vector forms. In this section we write the three systems of Sect. 3.2 in quaternion forms. The use of the quaternion forms of the function and the Cauchy-Riemann operator is called the biaxial quaternion analysis.

Consider the Cauchy-Riemann operator $D_{x}$ and the function $f: \mathbb{R}^{8} \rightarrow$ (1) in the quaternion forms:

$$
\begin{aligned}
D_{x} & =\partial_{u_{0}}+\partial_{\underline{u}}+\left(\partial_{v_{0}}+\partial_{\underline{v}}\right) \circ e_{4}, \\
f & =g_{0}+\underline{g}+\left(h_{0}+\underline{h}\right) \circ e_{4} .
\end{aligned}
$$

According to Corollary 2.4, we can write

$$
\begin{aligned}
D_{x} f= & \partial_{u_{0}} g_{0}-\partial_{v_{0}} h_{0}-\partial_{\underline{u}} \cdot \underline{g}-\partial_{\underline{v}} \cdot \underline{h} \\
& +\partial_{u_{0}} \underline{g}+\partial_{\underline{u}} g_{0}+\partial_{v_{0}} \underline{h}-\partial_{\underline{v}} h_{0}+\partial_{\underline{u}} \times \underline{g}-\partial_{\underline{v}} \times \underline{h} \\
& +\left(\partial_{u_{0}} h_{0}+\partial_{v_{0}} g_{0}+\partial_{\underline{v}} \cdot \underline{g}-\partial_{\underline{u}} \cdot \underline{h}\right) e_{4} \\
& +\left(\partial_{u_{0}} \underline{h}+\partial_{\underline{v}} g_{0}+\partial_{\underline{u}} h_{0}-\partial_{v_{0}} \underline{g}-\partial_{\underline{u}} \times \underline{h}-\partial_{\underline{v}} \times \underline{g}\right) \circ e_{4} .
\end{aligned}
$$

This implies the quaternion forms (14) and (15) of, respectively, the Moisil-Teodorescu type system (8) and the system (10).

Proposition 4.1. $f: \mathbb{R}^{8} \rightarrow \mathbb{O}$ is left regular if and only if it satisfies the system

$$
\begin{aligned}
& \partial_{u_{0}} h_{0}+\partial_{v_{0}} g_{0}+\partial_{\underline{v}} \cdot \underline{g}-\partial_{\underline{u}} \cdot \underline{h}=0, \\
& \partial_{u_{0}} g_{0}-\partial_{v_{0}} h_{0}-\partial_{\underline{u}} \cdot \underline{g}-\partial_{\underline{v}} \cdot \underline{h}=0, \\
& \partial_{u_{0}} \underline{g}+\partial_{\underline{u}} g_{0}+\partial_{v_{0}} \underline{h}-\partial_{\underline{v}} h_{0}+\partial_{\underline{u}} \times \underline{g}-\partial_{\underline{v}} \times \underline{h}=0, \\
& \partial_{u_{0}} \underline{h}+\partial_{\underline{v}} g_{0}+\partial_{\underline{u}} h_{0}-\partial_{v_{0}} \underline{g}-\partial_{\underline{u}} \times \underline{h}-\partial_{\underline{v}} \times \underline{g}=0 .
\end{aligned}
$$

Proposition 4.2. $f: \mathbb{R}^{8} \rightarrow \mathbb{O}$ is left and right regular if and only if it satisfies the system 


$$
\begin{aligned}
& \partial_{u_{0}} h_{0}+\partial_{v_{0}} g_{0}+\partial_{\underline{v}} \cdot \underline{g}-\partial_{\underline{u}} \cdot \underline{h}=0, \\
& \partial_{u_{0}} g_{0}-\partial_{v_{0}} h_{0}-\partial_{\underline{u}} \cdot \underline{g}-\partial_{\underline{v}} \cdot \underline{h}=0, \\
& \partial_{u_{0}} \underline{g}+\partial_{\underline{u}} g_{0}+\partial_{v_{0}} \underline{h}-\partial_{\underline{v}} h_{0}=0, \\
& \partial_{u_{0}} \underline{h}+\partial_{\underline{v}} g_{0}+\partial_{\underline{u}} h_{0}-\partial_{v_{0}} \underline{g}=0, \\
& \partial_{\underline{u}} \times \underline{g}-\partial_{\underline{v}} \times \underline{h}=0, \\
& \partial_{\underline{u}} \times \underline{h}+\partial_{\underline{v}} \times \underline{g}=0 .
\end{aligned}
$$

One example of the use of biaxial quaternion analysis is the proof of the following vector calculus identity in the octonionic case.

Lemma 4.3. Let the coordinates of $f: \mathbb{R}^{8} \rightarrow \mathbb{O}$ and $g: \mathbb{R}^{8} \rightarrow \mathbb{O}$ have partial derivatives. Then

$$
D_{\underline{x}} \cdot(\underline{f} \times \underline{g})=\left(D_{\underline{x}} \times \underline{f}\right) \cdot \underline{g}-\underline{f} \cdot\left(D_{\underline{x}} \times \underline{g}\right) .
$$

Proof. We use quaternion decompositions

$$
\begin{aligned}
D_{\underline{x}} & =\partial_{\underline{u}}+\partial_{v_{0}} e_{4}+\partial_{\underline{v}} \circ e_{4}, \\
\underline{f} & =\underline{f_{1}}+F_{0} e_{4}+\underline{F_{1}} \circ e_{4}, \\
\underline{g} & =\underline{g_{1}}+G_{0} e_{4}+\underline{G_{1}} \circ e_{4} .
\end{aligned}
$$

On the left-hand side we apply Lemma 2.3 to the cross product $f \times g$, and use the classical vector calculus identity

$$
\nabla \cdot(\underline{u} \times \underline{v})=(\nabla \times \underline{u}) \cdot \underline{v}-\underline{u} \cdot(\nabla \times \underline{v})
$$

for $\underline{u}, \underline{v}: \mathbb{R}^{3} \rightarrow \mathbb{R}^{3}$ :

$$
\begin{aligned}
D_{\underline{x}} \cdot(\underline{f} \times \underline{g})= & \left(\partial_{\underline{u}}+\partial_{v_{0}} e_{4}+\partial_{\underline{v}} \circ e_{4}\right) \cdot\left(F_{0} \underline{G_{1}}-\underline{F_{1}} G_{0}+\underline{f_{1}} \times \underline{g_{1}}-\underline{F_{1}} \times \underline{G_{1}}\right. \\
& +\left(\underline{F_{1}} \cdot \underline{g_{1}}-\underline{f_{1}} \cdot \underline{G_{1}}\right) e_{4} \\
& \left.+\left(\underline{f_{1}} G_{0}-F_{0} \underline{g_{1}}-\underline{f_{1}} \times \underline{G_{1}}-\underline{F_{1}} \times \underline{g_{1}}\right) \circ e_{4}\right) \\
= & \left.\partial_{\underline{u}} \cdot\left(F_{0} \underline{G_{1}}\right)-\partial_{\underline{u}} \cdot \underline{F_{1}} G_{0}\right)+\partial_{\underline{u}} \cdot\left(\underline{f_{1}} \times \underline{g_{1}}\right)-\partial_{\underline{u}} \cdot\left(\underline{F_{1}} \times \underline{G_{1}}\right) \\
& +\partial_{v_{0}}\left(\underline{F_{1}} \cdot \underline{g_{1}}\right)-\partial_{v_{0}}\left(\underline{f_{1}} \cdot \underline{G_{1}}\right) \\
& \left.+\partial_{\underline{v}} \cdot\left(\underline{f_{1}} G_{0}\right)-\partial_{\underline{v}} \cdot\left(F_{0} \underline{g_{1}}\right)-\partial_{\underline{v}} \cdot \underline{\left(f_{1}\right.} \times \underline{G_{1}}\right)-\partial_{\underline{v}} \cdot\left(\underline{F_{1}} \times \underline{g_{1}}\right) \\
= & \left(\partial_{\underline{u}} F_{0}\right) \cdot \underline{G_{1}}+F_{0}\left(\partial_{\underline{u}} \cdot \underline{G_{1}}\right)-\left(\partial_{\underline{u}} \cdot \underline{F_{1}}\right) G_{0}-\underline{F_{1}} \cdot\left(\partial_{\underline{u}} G_{0}\right) \\
& +\left(\partial_{\underline{u}} \times \underline{f_{1}}\right) \cdot \underline{g_{1}}-\underline{f_{1}} \cdot\left(\partial_{\underline{u}} \times \underline{g_{1}}\right)-\left(\partial_{\underline{u}} \times \underline{F_{1}}\right) \cdot \underline{G_{1}}+\underline{F_{1}} \cdot\left(\partial_{\underline{u}} \times \underline{G_{1}}\right) \\
& +\left(\partial_{v_{0}} \underline{F_{1}}\right) \cdot \underline{g_{1}}+\underline{F_{1}} \cdot\left(\partial_{v_{0}} \underline{g_{1}}\right)-\left(\partial_{v_{0}} \underline{f_{1}}\right) \cdot \underline{G_{1}}-\underline{f_{1}} \cdot\left(\partial_{v_{0}} \underline{G_{1}}\right) \\
& +\left(\partial_{\underline{v}} \cdot \underline{f_{1}}\right) G_{0}+\underline{f_{1}} \cdot\left(\partial_{\underline{v}} G_{0}\right)-\left(\partial_{\underline{v}} F_{0}\right) \cdot \underline{g_{1}}-F_{0}\left(\partial_{\underline{v}} \cdot \underline{g_{1}}\right) \\
& -\left(\partial_{\underline{v}} \times \underline{f_{1}}\right) \cdot \underline{G_{1}}+\underline{f_{1}} \cdot\left(\partial_{\underline{v}} \times \underline{G_{1}}\right)-\left(\partial_{\underline{v}} \times \underline{F_{1}}\right) \cdot \underline{g_{1}}+\underline{F_{1}} \cdot\left(\partial_{\underline{v}} \times \underline{g_{1}}\right) .
\end{aligned}
$$

On the right-hand side we apply Lemma 2.3 to the rotors $D_{\underline{x}} \times \underline{f}$ and $D_{\underline{x}} \times \underline{g}$ :

$$
\begin{aligned}
\left(D_{\underline{x}} \times \underline{f}\right) \cdot \underline{g}= & \left(\partial_{v_{0}} \underline{F_{1}}-\partial_{\underline{v}} F_{0}+\partial_{\underline{u}} \times \underline{f_{1}}-\partial_{\underline{v}} \times \underline{F_{1}}\right. \\
& +\left(\partial_{\underline{v}} \cdot \underline{f_{1}}-\partial_{\underline{u}} \cdot \underline{F_{1}}\right) e_{4} \\
& \left.+\left(\partial_{\underline{u}} F_{0}-\partial_{v_{0}} \underline{f_{1}}-\partial_{\underline{u}} \times \underline{F_{1}}-\partial_{\underline{v}} \times \underline{f_{1}}\right) \circ e_{4}\right) \cdot \\
& \left(\underline{g_{1}}+G_{0} e_{4}+\underline{G_{1}} \circ e_{4}\right) \\
= & \left(\partial_{v_{0}} \underline{F_{1}}\right) \cdot \underline{g_{1}}-\left(\partial_{\underline{v}} F_{0}\right) \cdot \underline{g_{1}}+\left(\partial_{\underline{u}} \times \underline{f_{1}}\right) \cdot \underline{g_{1}}-\left(\partial_{\underline{v}} \times \underline{F_{1}}\right) \cdot \underline{g_{1}}
\end{aligned}
$$




$$
\begin{aligned}
& +\left(\partial_{\underline{v}} \cdot \underline{f_{1}}\right) G_{0}-\left(\partial_{\underline{u}} \cdot \underline{F_{1}}\right) G_{0} \\
& +\left(\partial_{\underline{u}} F_{0}\right) \cdot \underline{G_{1}}-\left(\partial_{v_{0}} \underline{f_{1}}\right) \cdot \underline{G_{1}}-\left(\partial_{\underline{u}} \times \underline{F_{1}}\right) \cdot \underline{G_{1}}-\left(\partial_{\underline{v}} \times \underline{f_{1}}\right) \cdot \underline{G_{1}},
\end{aligned}
$$

and

$$
\begin{aligned}
\underline{f} \cdot\left(D_{\underline{x}} \times \underline{g}\right)= & \left(\underline{f_{1}}+F_{0} e_{4}+\underline{F_{1}} \circ e_{4}\right) . \\
& \left(\partial_{v_{0}} \underline{G_{1}}-\partial_{\underline{v}} G_{0}+\partial_{\underline{u}} \times \underline{g_{1}}-\partial_{\underline{v}} \times \underline{G_{1}}\right. \\
& +\left(\partial_{\underline{v}} \cdot \underline{g_{1}}-\partial_{\underline{u}} \cdot \underline{G_{1}}\right) e_{4} \\
& \left.+\left(\partial_{\underline{u}} G_{0}-\partial_{v_{0}} \underline{g_{1}}-\partial_{\underline{u}} \times \underline{G_{1}}-\partial_{\underline{v}} \times \underline{g_{1}}\right) \circ e_{4}\right) \\
= & \underline{f_{1}} \cdot\left(\partial_{v_{0}} \underline{G_{1}}\right)-\underline{f_{1}} \cdot\left(\partial_{\underline{v}} G_{0}\right)+\underline{f_{1}} \cdot\left(\partial_{\underline{u}} \times \underline{g_{1}}\right)-\underline{f_{1}} \cdot\left(\partial_{\underline{v}} \times \underline{G_{1}}\right) \\
& +F_{0}\left(\partial_{\underline{v}} \cdot \underline{g_{1}}\right)-F_{0}\left(\partial_{\underline{u}} \cdot \underline{G_{1}}\right) \\
& +\underline{F_{1}} \cdot\left(\partial_{\underline{u}} G_{0}\right)-\underline{F_{1}} \cdot\left(\partial_{v_{0}} \underline{g_{1}}\right)-\underline{F_{1}} \cdot\left(\partial_{\underline{u}} \times \underline{G_{1}}\right)-\underline{F_{1}} \cdot\left(\partial_{\underline{v}} \times \underline{g_{1}}\right) .
\end{aligned}
$$

Remark 4.4. (The set of Regular functions is not a module) In quaternion analysis $\partial_{u} g=0$ implies $\partial_{u}(g \circ a)=0$ for all $a \in \mathbb{H}$ (see Lemma 4.5). The same does not hold in octonion analysis. For example, define $g: \mathbb{H} \rightarrow \mathbb{H}$, $g(x)=x_{1}-x_{2} e_{3}$. Then $D_{x} g=e_{1}-e_{2} e_{3}=0$, but $D_{x}\left(g \circ e_{4}\right)=D_{x}\left(x_{1} e_{4}-\right.$ $\left.x_{2} e_{7}\right)=e_{1} e_{4}-e_{2} e_{7}=2 e_{5}$.

For quaternion functions we have the product rules (16) and (17) for the Cauchy-Riemann operator. Remark 4.4 suggests that we do not have any kind of a non-trivial product rule for octonion valued functions. In practice, one way to compute $D_{x}(f g)$ for octonion valued functions is to use biaxial quaternion analysis, and then to apply (16)-(24).

Lemma 4.5. ([3, Thm 1.3.2]) Let the coordinates of $f: \mathbb{H} \rightarrow \mathbb{H}$ and $g: \mathbb{H} \rightarrow \mathbb{H}$ have partial derivatives. Then

$$
\partial_{\underline{u}}(f \circ g)=\left(\partial_{\underline{u}} f\right) \circ g+\bar{f} \circ\left(\partial_{\underline{u}} g\right)-2\left(\underline{f} \cdot \partial_{\underline{u}}\right) g
$$

and

$$
(f \circ g) \partial_{\underline{u}}=\left(f \partial_{\underline{u}}\right) \circ \bar{g}+f \circ\left(g \partial_{\underline{u}}\right)-2\left(\underline{g} \cdot \partial_{\underline{u}}\right) f .
$$

Here, $\left(\underline{f} \cdot \partial_{\underline{u}}\right) g=\sum_{i=1}^{3} f_{i} \partial_{x_{i}} g$.

Corollary 4.6. Let the coordinates of $f: \mathbb{H} \rightarrow \mathbb{H}$ and $g: \mathbb{H} \rightarrow \mathbb{H}$ have partial derivatives. Then

$$
\begin{aligned}
\partial_{\underline{u}}\left(\left(f \circ e_{4}\right) \circ g\right) & =\left[\left(f \partial_{\underline{u}}\right) \circ g+f \circ\left(\bar{g} \partial_{\underline{u}}\right)+2\left(\underline{g} \cdot \partial_{\underline{u}}\right) f\right] \circ e_{4} \\
\partial_{\underline{u}}\left(f \circ\left(g \circ e_{4}\right)\right) & =\left[\left(g \partial_{\underline{u}}\right) \circ \bar{f}+g \circ\left(f \partial_{\underline{u}}\right)-2\left(\underline{f} \cdot \partial_{\underline{u}}\right) g\right] \circ e_{4} \\
\partial_{\underline{u}}\left(\left(f \circ e_{4}\right) \circ\left(g \circ e_{4}\right)\right) & =-\left(\partial_{\underline{u}} \bar{g}\right) \circ f-g \circ\left(\partial_{\underline{u}} f\right)-2\left(\underline{g} \cdot \partial_{\underline{u}}\right) f \\
\left(\partial_{\underline{v}} \circ e_{4}\right)(f \circ g) & =\left[\left(\partial_{\underline{v}} \bar{g}\right) \circ \bar{f}+g \circ\left(\partial_{\underline{v}} \bar{f}\right)+2\left(\underline{g} \cdot \partial_{\underline{v}}\right) \bar{f}\right] \circ e_{4} \\
\left(\partial_{\underline{v}} \circ e_{4}\right)\left(\left(f \circ e_{4}\right) \circ g\right) & =-\left(g \partial_{\underline{v}}\right) \circ f-g\left(\bar{f} \partial_{\underline{v}}\right)-2\left(\underline{f} \cdot \partial_{\underline{v}}\right) g \\
\left(\partial_{\underline{v}} \circ e_{4}\right)\left(f \circ\left(g \circ e_{4}\right)\right) & =-\left(\bar{f} \partial_{\underline{v}}\right) \circ g-\bar{f} \circ\left(\bar{g} \partial_{\underline{v}}\right)-2\left(\underline{g} \cdot \partial_{\underline{v}}\right) \bar{f} \\
\left(\partial_{\underline{v}} \circ e_{4}\right)\left(\left(f \circ e_{4}\right) \circ\left(g \circ e_{4}\right)\right) & =\left[-\left(\partial_{\underline{v}} \bar{f}\right) g-f\left(\partial_{\underline{v}} g\right)-2\left(\underline{f} \cdot \partial_{\underline{v}}\right) g\right] \circ e_{4}
\end{aligned}
$$

Proof. Apply Lemmas 2.1 and 4.5, and use the fact $\overline{f g}=\bar{g} \bar{f}$. 


\section{Function Classes in Clifford Analysis}

In this last section, we study the classes of left-, $B$-, and $R$-regular functions using Clifford analysis. We begin with the following algebraic lemma.

Lemma 5.1. Let I be the primitive idempotent

$$
I=\frac{1}{16}\left(1+W e_{12 \cdots 7}\right)\left(1-e_{12 \cdots 7}\right),
$$

where $W=e_{123}+e_{145}+e_{176}+e_{246}+e_{257}+e_{347}+e_{365}$, and let $a=a_{0}+\underline{a}$ and $b=b_{0}+\underline{b} \in \mathcal{C} \ell_{0,7}$ be paravectors. Then

$$
\begin{gathered}
16[a b I]_{0}=a_{0} b_{0}-\underline{a} \cdot \underline{b}, \\
16[a b I]_{1}=a_{0} \underline{b}+\underline{a} b_{0}-[(\underline{a} \wedge \underline{b}) W]_{1}, \\
16[a b I]_{2}=\underline{a} \wedge \underline{b}-\left[\left(a_{0} \underline{b}+\underline{a} b_{0}\right) W\right]_{2}+\left[(\underline{a} \wedge \underline{b}) W e_{1 \cdots 7}\right]_{2}, \\
16[a b I]_{3}=-\left(a_{0} b_{0}-\underline{a} \cdot \underline{b}\right) W+\left[\left(a_{0} \underline{b}+\underline{a} b_{0}\right) W e_{1 \cdots 7}\right]_{3}-[(\underline{a} \wedge \underline{b}) W]_{3}, \\
16[a b I]_{4}=\left(a_{0} b_{0}-\underline{a} \cdot \underline{b}\right) W e_{1 \cdots 7}-\left[\left(a_{0} \underline{b}+\underline{a} b_{0}\right) W\right]_{4}+\left[(\underline{a} \wedge \underline{b}) W e_{1 \cdots 7}\right]_{4}, \\
16[a b I]_{5}=\left[\left(a_{0} \underline{b}+\underline{a} b_{0}\right) W e_{1 \cdots 7}\right]_{5}-[(\underline{a} \wedge \underline{b}) W]_{5}-(\underline{a} \wedge \underline{b}) e_{1 \cdots 7}, \\
16[a b I]_{6}=-\left(a_{0} \underline{b}+\underline{a} b_{0}\right) e_{1 \cdots 7}+\left[(\underline{a} \wedge \underline{b}) W e_{1 \cdots 7}\right]_{6}, \\
16[a b I]_{7}=-\left(a_{0} b_{0}-\underline{a} \cdot \underline{b}\right) e_{1 \cdots 7},
\end{gathered}
$$

and

$$
[a b I]_{k}=0 \Leftrightarrow[a b I]_{7-k}=0, \quad k=0,1, \ldots, 7 .
$$

If $[a b I]_{0}=0$, then the conditions $[a b I]_{j}=0, j=2,3,4,5$, are pairwise equivalent. In particular, if $[a b I]_{0,1,2}=0$, then $a b I=0$.

Proof. Write the real part and the 1- and 2-vector parts of $a b$ using (3), and expand the definition (4) of $I$ using the fact $e_{12 \cdots 7}^{2}=1$ :

$$
\begin{aligned}
a b & =\left(a_{0} b_{0}-\underline{a} \cdot \underline{b}\right)+\left(a_{0} \underline{b}+\underline{a} b_{0}\right)+\underline{a} \wedge \underline{b}, \\
16 I & =1-W+W e_{12 \cdots 7}-e_{12 \cdots 7} .
\end{aligned}
$$

Here, $W$ is a 3 -vector and $W e_{12 \cdots 7}$ is a 4 -vector. Then, for example, $\underline{a} W$ only contains 2 - and 4 -vector parts, and therefore $[\underline{a} W]_{3}=0$. This kind of reasoning implies $(25)-(32)$.

Now, (33) follows from the facts that for any $c \in \mathcal{C} \ell_{0,7}$,

$$
\begin{aligned}
& c=0 \Leftrightarrow c e_{12 \cdots 7}=0, \quad \text { and } \\
& {[c]_{k} e_{12 \cdots 7}=\left[c e_{12 \cdots 7}\right]_{7-k}, \quad k=0,1, \ldots, 7 .}
\end{aligned}
$$

To prove the last claim, it is now enough to show that in the case $[a b I]_{0}=0$, $[a b I]_{2}=0$ if and only if $[a b I]_{3}=0$. This can be seen by computing

$$
\begin{aligned}
16[a b I]_{2}= & \left(a_{0} b_{1}+a_{1} b_{0}+a_{2} b_{3}-a_{3} b_{2}+a_{4} b_{5}-a_{5} b_{4}\right. \\
& \left.-a_{6} b_{7}+a_{7} b_{6}\right)\left(e_{23}+e_{45}-e_{67}\right) \\
& +\left(a_{0} b_{2}-a_{1} b_{3}+a_{2} b_{0}+a_{3} b_{1}+a_{4} b_{6}\right. \\
& \left.+a_{5} b_{7}-a_{6} b_{4}-a_{7} b_{5}\right)\left(-e_{13}+e_{46}+e_{57}\right) \\
& +\left(a_{0} b_{3}+a_{1} b_{2}-a_{2} b_{1}+a_{3} b_{0}\right. \\
& \left.+a_{4} b_{7}-a_{5} b_{6}+a_{6} b_{5}-a_{7} b_{4}\right)\left(e_{12}+e_{47}-e_{56}\right)
\end{aligned}
$$




$$
\begin{aligned}
& +\left(a_{0} b_{4}-a_{1} b_{5}-a_{2} b_{6}-a_{3} b_{7}\right. \\
& \left.+a_{4} b_{0}+a_{5} b_{1}+a_{6} b_{2}+a_{7} b_{3}\right)\left(-e_{15}-e_{26}-e_{37}\right) \\
& +\left(a_{0} b_{5}+a_{1} b_{4}-a_{2} b_{7}+a_{3} b_{6}\right. \\
& \left.-a_{4} b_{1}+a_{5} b_{0}-a_{6} b_{3}+a_{7} b_{2}\right)\left(e_{14}-e_{27} e_{36}\right) \\
& +\left(a_{0} b_{6}+a_{1} b_{7}+a_{2} b_{4}-a_{3} b_{5}\right. \\
& \left.-a_{4} b_{2}+a_{5} b_{3}+a_{6} b_{0}-a_{7} b_{1}\right)\left(e_{17}+e_{24}-e_{35}\right) \\
& +\left(a_{0} b_{7}-a_{1} b_{6}+a_{2} b_{5}+a_{3} b_{4}-a_{4} b_{3}\right. \\
& \left.-a_{5} b_{2}+a_{6} b_{1}+a_{7} b_{0}\right)\left(-e_{16}+e_{25}+e_{34}\right)
\end{aligned}
$$

and in the case $[a b I]_{0}=0$,

$$
\begin{aligned}
16[a b I]_{3}= & \left(a_{0} b_{1}+a_{1} b_{0}+a_{2} b_{3}-a_{3} b_{2}+a_{4} b_{5}-a_{5} b_{4}\right. \\
& \left.-a_{6} b_{7}+a_{7} b_{6}\right)\left(e_{247}-e_{256}-e_{346}-e_{357}\right) \\
& +\left(a_{0} b_{2}-a_{1} b_{3}+a_{2} b_{0}+a_{3} b_{1}+a_{4} b_{6}\right. \\
& \left.+a_{5} b_{7}-a_{6} b_{4}-a_{7} b_{5}\right)\left(-e_{147}+e_{156}+e_{345}-e_{367}\right) \\
& +\left(a_{0} b_{3}+a_{1} b_{2}-a_{2} b_{1}+a_{3} b_{0}\right. \\
& \left.+a_{4} b_{7}-a_{5} b_{6}+a_{6} b_{5}-a_{7} b_{4}\right)\left(e_{146}+e_{157}-e_{245}+e_{267}\right) \\
& +\left(a_{0} b_{4}-a_{1} b_{5}-a_{2} b_{6}-a_{3} b_{7}+a_{4} b_{0}\right. \\
& \left.+a_{5} b_{1}+a_{6} b_{2}+a_{7} b_{3}\right)\left(e_{127}-e_{136}+e_{235}-e_{567}\right) \\
& +\left(a_{0} b_{5}+a_{1} b_{4}-a_{2} b_{7}+a_{3} b_{6}-a_{4} b_{1}\right. \\
& \left.+a_{5} b_{0}-a_{6} b_{3}+a_{7} b_{2}\right)\left(-e_{126}-e_{137}-e_{234}+e_{467}\right) \\
& +\left(a_{0} b_{6}+a_{1} b_{7}+a_{2} b_{4}-a_{3} b_{5}-a_{4} b_{2}\right. \\
& \left.+a_{5} b_{3}+a_{6} b_{0}-a_{7} b_{1}\right)\left(e_{125}+e_{134}-e_{237}-e_{457}\right) \\
& +\left(a_{0} b_{7}-a_{1} b_{6}+a_{2} b_{5}+a_{3} b_{4}-a_{4} b_{3}\right. \\
& \left.-a_{5} b_{2}+a_{6} b_{1}+a_{7} b_{0}\right)\left(-e_{124}+e_{135}+e_{236}+e_{456}\right) .
\end{aligned}
$$

We infer that left-, B-, and R-regularity can be studied by considering paravector-spinor valued functions $f I$.

Theorem 5.2. Suppose that $f: \mathbb{R}^{8} \rightarrow \mathbb{R}^{8}$ is a paravector valued function such that the coordinate functions have partial derivatives.

(a) $f$ is left-regular if and only if

$$
\left[\partial_{x} f I\right]_{j}=0 \text { for } j=0,1 .
$$

(b) $f$ is B-regular if and only if

$$
\left[\partial_{x} f I\right]_{j}=0 \text { for } j=0,1, \quad \text { and } \quad\left[\partial_{x} f W\right]_{1}=0 .
$$

Proof. (a) follows using Lemma 2.7:

$$
D_{x} f=16\left[\partial_{x} f I\right]_{0}+16\left[\partial_{x} f I\right]_{1} .
$$


(b) From (3) and (26), we obtain

$$
\begin{aligned}
{\left[\partial_{x} f W\right]_{1} } & =\left[\left(\partial_{x_{0}} f_{0}-\partial_{\underline{x}} \cdot \underline{f}\right) W\right]_{1}+\left[\left(\partial_{x_{0}} \underline{f}+\partial_{\underline{x}} f_{0}\right) W\right]_{1}+\left[\left(\partial_{\underline{x}} \wedge \underline{f}\right) W\right]_{1} \\
& =\left[\left(\partial_{\underline{x}} \wedge \underline{f}\right) W\right]_{1} \\
& =-16\left[\partial_{x} f I\right]_{1}+\partial_{x_{0}} \underline{f}+D_{\underline{x}} f_{0} .
\end{aligned}
$$

Since $D_{\underline{x}} \times \underline{f}=-\left[\left(\partial_{\underline{x}} \wedge \underline{f}\right) W\right]_{1}$ (Lemma 2.5), the claim now follows from (a) and Propositions 3.1-3.2.

Remark 5.3. If $\partial_{x} f=0$, then (trivially) $\left[\partial_{x} f I\right]_{j}=0$ for all $j=0,1, \ldots, 7$. The converse does not hold. This follows from the fact that the equation $a I=0$ does not have a unique solution $a=0$ in the Clifford algebra. Hence, paravector spinor valued solutions of the Cauchy-Riemann equations form a bigger function class, and the class of R-regular solutions is

$$
\begin{aligned}
& \mathcal{M}_{R} \subsetneq\left\{f: \partial_{x} f I=0\right\}=\left\{f:\left[\partial_{x} f I\right]_{j}=0, j=0,1, \ldots, 7\right\} \\
& \quad=\left\{f:\left[\partial_{x} f I\right]_{j}=0, j=0,1,2\right\} .
\end{aligned}
$$

The equality of the latter two function classes follows from Lemma 5.1. An example showing that the inclusion is strict: if $f=x_{2} e_{1}-x_{7} e_{4}$, then $\partial_{x} f=$ $e_{4} e_{7}-e_{1} e_{2}$, but $\left[\partial_{x} f I\right]_{j}=0$ for $j=0,1,2$.

\section{Conclusion}

The key idea of this paper is to study differences between octonion and Clifford analyses. This leads us to observe the fundamental difference between octonion regular and Clifford monogenic functions. The structure of octonion regular functions is studied by comparing left-, right-, $B$-, and $R$-regular functions. The existence of these classes is a consequence of different algebraic properties of the algebras. In the heart of octonion analysis is the study of the properties of these function classes and their relations, which distinguishes it essentially from Clifford analysis.

Open Access. This article is distributed under the terms of the Creative Commons Attribution 4.0 International License (http://creativecommons.org/licenses/by/4. $0 /$, which permits unrestricted use, distribution, and reproduction in any medium, provided you give appropriate credit to the original author(s) and the source, provide a link to the Creative Commons license, and indicate if changes were made.

Publisher's Note Springer Nature remains neutral with regard to jurisdictional claims in published maps and institutional affiliations.

\section{References}

[1] Delanghe, R., Sommen, F., Souček, V.: Clifford Algebra and Spinor-Valued Functions. Mathematics and its Applications. Kluwer Academic Publishers Group, Dordrecht (1992) 
[2] Dentoni, P., Sce, M.: Funzioni regolari nell'algebra di Cayley. Rend. Semin. Mat. Univ. Padova 50, 251-267 (1973)

[3] Gürlebeck, K., Sprössig, W.: Quaternionic Analysis and Elliptic Boundary Value Problems. International Series of Numerical Mathematics. Birkhäuser Verlag, Basel (1990)

[4] Harvey, F.: Spinors and Calibrations. Perspectives in Mathematics. Academic Press, Boston (1990)

[5] Kauhanen, J., Orelma, H.: Cauchy-Riemann operators in octonionic analysis. Adv. Appl. Clifford Algebr. 28(1) (2018), Art. 1

[6] Kauhanen, J., Orelma, H.: Some theoretical remarks of octonionic analysis. AIP Conf. Proc. 1907(1), 5 (2017)

[7] Li, X., Peng, L.: On Stein-Weiss conjugate harmonic function and octonion analytic function. Approx. Theory Appl. (N.S.) 16(2), 28-36 (2000)

[8] Lounesto, P.: Clifford Algebras and Spinors, second edition. London Mathematical Society Lecture Note Series, 286. Cambridge University Press, Cambridge (2001)

[9] Peng, L., Yang, L.: The curl in seven dimensional space and its applications. Approx. Theory Appl. (N.S.) 15(3), 66-80 (1999)

[10] Porteous, I.: Clifford Algebras and the Classical Groups. Cambridge Studies in Advanced Mathematics (Book 50). Cambridge University Press, Cambridge (1995)

[11] Study, E.: Geometrie der Dynamen. Die Zusammensetzung von Kräften und verwandte Gegenstände der Geometrie. Teubner, Leipzig (1903)

[12] Venäläinen, A.: On octonionic analysis. Licentiate thesis, University of Joensuu (2004)

Janne Kauhanen

Mathematics

Tampere University

33014 Tampere

Finland

e-mail: janne.kauhanen@tuni.fi

Heikki Orelma

Civil Engineering

Tampere University

33014 Tampere

Finland

e-mail: heikki.orelma@tuni.fi

Received: January 9, 2019.

Accepted: June 20, 2019. 